\title{
ИГРОФИКАЦИЯ ПРОЦЕССА РЕШЕНИЯ ТИПОВЫХ УЧЕБНЫХ ЗАДАЧ НА ОСНОВЕ ВЫБОРА ПРАВИЛ ПРЕОБРАЗОВАНИЯ
}

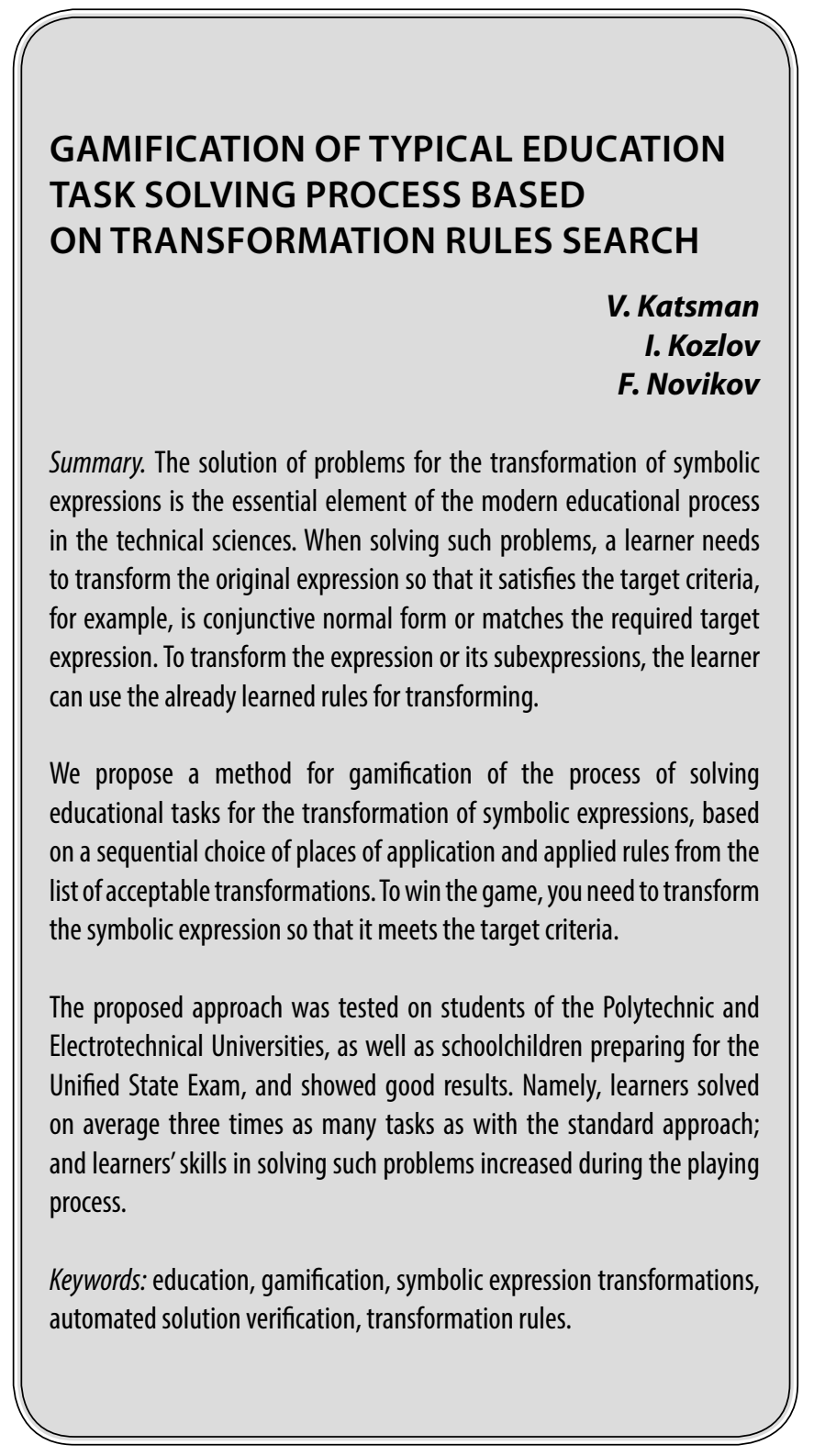

\section{Введение}

$\mathbf{H}$ а сегодняшний день вопросы стимулирования обучающихся, повышения результативности обучения и эффективности труда преподавателей, а также доступности образования более чем актуальны. Системы автоматизации обучения становятся все более популярными, они позволяют существенно упростить работу преподавателя и масштабировать ее для большого числа обучаемых. Преподавателям требуется разово,
Качман Виктор Игоревич

Аспирант, Санкт-Петербургский Политехнический Университет Петра Великого vikto9494@gmail.com

Козлов Илья Алексеевич

Санкт-Петербургский Политехнический Университет Петра Великого

kozlovilya@bk.ru

Новиков Федор Александрович

Д.т.н., профессор, Санкт-Петербургский Политехнический Университет Петра Великого fedornovikov51@gmail.com

Аннотация. Одним из основных элементов современного образовательного процесса в области технических наук является решение задач на преобразование символьных выражений. При решении таких задач учащимся требуется преобразовать исходное выражение так, чтобы оно удовлетворяло целевым критериям, например, являлось конъюнктивной нормальной формой или совпадало с требуемым целевым выражением. Для совершения преобразований над выражением или его подвыражениями учащийся может использовать уже изученные правила преобразования.

Мы предлагаем способ игрофикации процесса решения учебных задач на преобразование символьных выражений, основанный на последовательном выборе мест применения и применяемых правил из списка допустимых преобразований. Для победы в игре необходимо преобразовать символьное выражение так, чтобы оно удовлетворяло целевым критериям.

Предложенный подход был опробован на студентах Политехнического и Электротехнического Университетов, а также готовящихся к ЕГЭ школьниках и дал неплохие результаты. А именно, учащиеся решили в среднем втрое большее число задач, чем при стандартном подходе; а навыки учащихся по решению подобных задач возрастали в ходе игры.

Ключевые слова: образование, игрофикация, преобразование символьных выражений, автоматическая проверка решений, правила преобразований.

но качественно продумать образовательную программу - и машины смогут донести ее до большого числа людей, качество их работы не будет зависеть от личных обстоятельств, а только от заложенного в машинах алгоритма [1-3].

После объяснения нового материала, необходимо добиться, чтобы обучаемый глубоко усвоил новую информацию. Для этого ему необходимо решить большое число задач. Это прежде всего влечет необходимость 
подбора задач, для решения которых требуется применение именно новых навыков, а также навыков, хуже всего усвоенных обучаемым в ходе уже прошедшего обучения. Кроме подбора задач, требуется также проверка их решений. Желательно, чтобы при получении результатов проверки, учащийся мог разобраться, где именно он мог допустить ошибку, а, может быть, еще и получить подсказку, как эту ошибку исправлять.

Помимо этих базовых вещей требуется стимулировать учащихся к добросовестному решению подобранных задач. Эта составляющая является особенно актуальной, поскольку самостоятельной мотивации у большинства обучаемых не хватает. Наиболее распространенные на сегодняшний день комбинации методов словесных внушений с последующим контролем также не подходят для существенной части обучаемых. Игровые методы подходят для стимулирования намного лучше, но, существуют лишь для очень небольшого числа узких типов учебных задач и плохо поддаются автоматизации.

В этой статье мы предлагаем способ игрофикации процесса решений типовых учебных задач на преобразование символьных выражений по изученным правилам или формулам. Примеры подобных задач есть в самых разных областях точных наук, например: доказать, что

$$
\frac{\sin (x)}{1+\cos (x)}+\frac{1+\cos (x)}{\sin (x)}=\frac{2}{\sin (x)}
$$

Привести к КНФ:

$$
\overline{x \wedge(y \backslash z)}
$$

вывести формулу зарфда $q$ через $A, I, R$.

Решение подобных задач необходимо для закрепления навыков уверенного владения формулами, которое является фундаментом для решения более творческих и практических задач. Соответственно, существенное повышение объема решаемых учащимися задач на знание формул будет способствовать повышению качества образовательного процесса.

Предлагаемый способ особенно хорошо подходит для задач по логическим исчислениям, базовым операциям над полиномами и тригонометрии. На основе предлагаемого подхода нами была реализована игра в виде приложения под Android. Разработанная игра была опробована на студентах младших курсов Политехнического и Электротехнического Университетов на задачах по логическим исчислениям и комбинаторике, а также на готовящихся к ЕГЭ школьниках и показала неплохие результаты.
Сушествуюшие по ххо ы

к игрофикашии

На текущий момент уже существует множество трудов с разнообразными подходами к игрофикации в образовании [4-7]. Многие из них уже активно и успешно используются. Они существенно различаются:

1. По цели применения: от привлечения внимания обучаемых к излагаемому преподавателем материалу - до стимулирования самостоятельной активности обучаемых, например, направленной на решение практических задач.

2. По связанности используемых игровых методов с предметной областью: от использования общепринятых привлекающих визуальных и игровых эффектов - до завязки игрового сюжета на предметную область и необходимости совершения стандартных при решении действий в игровом формате. Например, при игре в «Евклюдию» требуется строить геометрические примитивы с помощью циркуля и линейки аналогично их построению на бумаге.

Эти подходы используют стандартные особенности обучающихся: сравнение по рейтингам [5], конкуренцию за в дальнейшем используемые ресурсы [6], любопытство, заинтересованность в неожиданных явлениях, положительную обратную связь [7]. Поясним описанную классификацию с помощью нескольких примеров.

Пример 1. На лекциях преподаватель использует презентации с красивыми картинками на слайдах, а в конце предлагает обучаемым угадывать, к какой теме какая картинка относилась. Данный подход может привлекать внимание обучаемых к материалу на слайдах преподавателя, но никак не связан с изучаемой предметной областью.

Пример 2. На слайдах презентации преподаватель использует фрагменты большой картины-пазла, которые обучаемым требуется состыковывать по принципу связанности материала на слайдах: чем больше смысловая связь между слайдами - тем больше вероятность, что фрагменты картины на этих слайдах смежны друг к другу. В этом примере игрофикация сильнее связанна с теоретическим материалом на слайдах, но у обучаемых остается возможность собрать пазл перебором без существенного вникания в предметную область.

Оба приведенных выше примера игрофикации поддаются автоматизации и масштабированию, легко могут быть реализованы в онлайн-курсе на современной платформе для систем дистанционного обучения [8-12]. Но они годятся преимущественно для подачи теорети- 
ческой части материала, из практических навыков они развивают лишь относящиеся к распознаванию картинок и сбору пазлов, что едва ли относится к областям технических наук.

Пример 3. Обучающимся предлагается одновременно решать практические задачи, тот, кто раньше введет в систему правильный ответ, получает бонус. Например, влияющий на итоговую оценку балл. Игры такого типа хорошо зарекомендовали себя и стимулируют учащихся решать полезные для предмета практические задачи, если они по силам учащихся. Существует множество платформ для создания подобных игр [13-14]. Но они не позволяют игрофицировать сам процесс решения задачи. Мотивация обучаемых растет исключительно за счет соревновательных эффектов, красивой графики и захватывающих анимаций.

Пример 4. Игры «Евклидия» [15], «Пифагория» [16], «XSection» [17]. В них требуется строить геометрические примитивы с помощью циркуля и линейки. Построение происходит на экране цифрового устройства, которое упрощает технические детали построения, не требует наличия личных и качественных циркуля и линейки, автоматически делает полученный чертеж красивым и приятным. При этом сам процесс решения идентичен процессу решения при стандартном процессе решения аналогичных задач, например, на бумаге. При этом, за счет более простой формы построения решения, добавляется возможность подачи обучаемым более сложных учебных задач [18]. То есть практикуются навыки решения стандартных и более сложных задач на построение, повышается уровень владения геометрическими примитивами, но в более приятной для обучающихся форме.

Помимо перечисленных «Евклидия», «Пифагория», «XSection», существует еще множество подобных игр, заточенных именно на игрофикацию самого процесса решения задачи, например, «GeoGebra» [19]. Подобные схемы игрофикации представляются наиболее продуктивными и интересными, их можно дополнить стандартными соревновательными и графическими эффектами. Однако проблема в том, что они на текущий момент подходят для очень небольшого числа узких областей, в число которых не входят логические исчисления, сложные формулы тригонометрии и другие.

Предлагаемый в статье подход существенно расширяет пространство возможностей игрофикации процесса решения учебных задач на задачи преобразования символьных выражений, практика решения которых необходима для качественного изучения почти всех областей точных наук.

\section{Роль правил преобразования в прошессе решения}

В процессе решения типовой учебной задачи на преобразование символьных выражений обучаемый первым делом читает и интерпретирует условие задачи, данное в нем стартовое выражение и цель, к которой его нужно свести. После этого обучаемый начинает шаг за шагом преобразовывать стартовое выражение и так происходит до тех пор, пока очередное преобразование не приводит его к решению задачи. В качестве решения задачи обучаемый предъявляет цепочку преобразований (шагов).

При проверке решения, такая цепочка соответствует процессу решения задачи после понимания её условия и оказывается единственной информацией, которую преподаватель получает для проверки. То есть, при проверке письменного решения преподаватель может проверить лишь корректность предъявляемой учащимся цепочки преобразований без возможности проверить ход рассуждений обучаемого при интерпретации условия - можно проверить только конечный результат: выражение мыслей обучаемого формулами. На основе данных идей нами был разработан метод перебора правил для автоматической проверки правильности цепочек преобразований [20-21]. Этот метод теоретически позволяет проверить решения типовых учебных задач, которые можно описать правилами, следующими из формул.

Рассмотрим, как выглядит процесс вывода решения задачи для обучаемого. Если обучаемый хорошо владеет формулами или правилами преобразований, для него процесс решения задачи также разбивается на шаги, в каждом из которых требуется выбрать одно из правил преобразования и место в выражении, где это правило преобразование нужно применить. Подобные действия повторяются пока исходное выражение не станет удовлетворять требуемым условиям.

В случае, если обучаемый плохо знает допустимые правила преобразования, ему приходится либо искать их в справочниках, либо выводить вручную, либо угадывать. Эти варианты подходят при решении относительно простых задач, но начинают отнимать слишком много времени при решении сложных, которые не решаются за маленькое число шагов или присутствуют шаги, на которых требуется делать нестандартные шаги (например, добавить и вычесть некоторое слагаемое), исходя из знаний о правилах, которые позволят прийти к решению после нестандартного шага.

Таким образом, простые задачи стимулируют обучаемого изучить существующие правила и начать их запоми- 


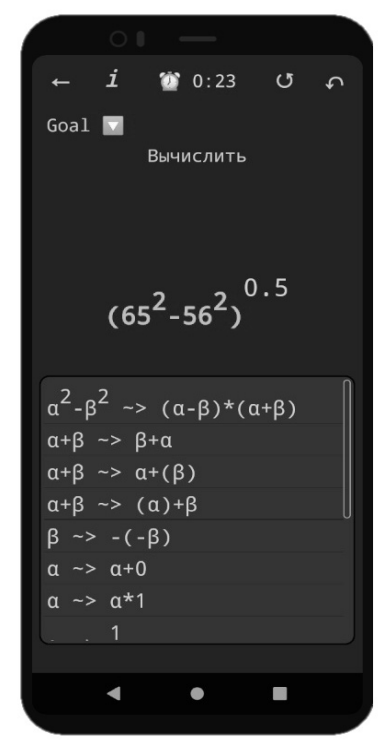

Рис. 1. Выбор места и правила преобразования

нать. Далее приходит время более сложных задач, в которых требуется владение правилами преобразования уже на более высоком уровне, требуется выстраивать стратегию их применения. Заметим, что решать простые задачи на этом этапе познаний обучаемым становится неинтересно, однократное или двукратное применение уже известных правил преобразований становится рутинным (например, как устный счет для людей, хорошо освоивших базовую арифметику).

\section{Премлагаемый способ игрофикашии}

Описанное в предыдущей секции почти напрямую конвертируется в способ игрофикации процесса решения задач на преобразование символьных выражений. Задачи соответствуют уровням; исходно данные выражения - стартовым конфигурациям уровней; выполнение требований к цели преобразований - целям, при выполнении которых уровни проходятся.

Вначале каждого уровня игрок получает стартовое выражение и цель - то, к чему его нужно свести. А именно, другое исходно заданное выражение (аналогично задачам на доказательство), либо выражение, удовлетворяющее определенным требованиям, например, 3-КНФ (аналогично задачам на сведение к 3-КНФ) или выражение без операций деления и отрицательных степеней (аналогично задачам на сокращение дробей). Стартовое выражение интерактивно, в него можно кликать и выделять различные подвыражения. Для каждого клика и выделенного подвыражения показываются применимые к этому подвыражению правила преобразований, игрок может кликнуть в любое из них - и оно автоматически применится к стартовому выражению.

Таким образом, на каждом шаге игрок сначала выбирает место преобразования в стартовом выражении, а затем применимое в этом месте правило преобразования (см рисунок 1). Стартовое выражение автоматически модифицируется по этому правилу, игрок вновь выбирает место и правило преобразования. Так продолжается до тех пор, пока стартовое выражение не станет удовлетворять целевым критериям. Тогда уровень становится пройденным, число шагов, за которое он пройден показывает, насколько хорошо игрок с ним справился.

Предлагаемый способ очень точно игрофицирует процесс решения учебных задач на преобразование символьных выражений для обучаемых, уверенно владеющих правилами преобразования в рассматриваемой предметной области. При незнании обучаемыми правил преобразования, игра подсказывает им, выводя исключительно возможные правила преобразования, из которых нужно лишь выбрать актуальное. На простых уровнях игроки могут изучать новые для них правила, а затем переходить к более сложным.

Здесь есть существенный нюанс, связанный с возможностью угадывания правил преобразования на несложных уровнях. А именно, игрок может угадыванием пройти легкие уровни, не изучив правил преобразования. Проходить без знания правил преобразования сложные уровни почти невозможно, угадывать приходится из слишком большого числа вариантов правил и мест на слишком большом количестве шагов. А это 
может приводить к разочарованию обучаемого, игра перестает быть интересной для него.

Поэтому предлагается на начальных простых уровнях давать задачи с небольшим количеством шагов, но с большим выбором правил преобразования, где игроку будет необходимо именно вникать в правила преобразования, угадывание должно становиться сложным.

Помимо игрофицирования процесса решения учебных задач на преобразование символьных выражений, предлагаемый подход позволяет автоматически проверять решения задач, ведь последовательность выбранных игроком правил и мест применения однозначно соответствует цепочке преобразований над символьными выражениями. Корректной, если все выбранные игроком правила правильны, и некорректной в противном случае.

Также игра существенно затрудняет списывание и заимствование результатов чужих трудов. Обмениваться последовательностями мест применения правил и самих правил преобразования, примененных в игре существенно сложнее, чем обмениваться ответами и даже решениями задач.

\section{Эксперименты и результаты}

Эксперименты проводились с целью убедиться, что предложенный способ игрофикации, во-первых, действительно стимулирует обучаемых к решению больших объемов задач. А во-вторых, действительно ли навыки учащихся возрастают в ходе подобных игр и как рост этих навыков при решении задач в игровом формате соотносится с ростом аналогичных навыков при решении задач в стандартной форме.

На основе предлагаемого метода нами было реализовано Android приложение с играми по описанной выше логике [22]. По воле случая, проводимые нами эксперименты пришлись на время самоизоляции; применение стандартных учебных мероприятий стало существенно затруднено, в связи с чем реализованные нами игры в Android-приложении использовались намного шире, чем мы рассчитывали.

По итогам, нами были проведены следующие эксперименты:

1. Студентам 2-го курса СПБПУ и студентам 1-го курса ЛЭТИ в рамках курса "дискретная математика" были предложены игры на логические исчисления и комбинаторные формулы. Первая игра включала в себя задачи на сведение одного логического выражения к другому, на метод резолю- ций и на сведение логических выражений к НФ, всего 36 уровней-задач. Вторая - задачи на числа размещений, сочетаний и разбиений, всего 13 уровней-задач.

2. Случайно отобранным желающим учащимся 10-11x классов были предложены игры со связанными с ЕГЭ по математике и физике задачами на темы «формулы сокращенного умножения», «иррациональность», «логарифм», «тригонометрия», «перемещение», «молекулярная физика», «электродинамика» и «квантовая физика».

В целом в проведенных экспериментах обучаемые решали в среднем втрое больше число учебных задач, чем при стандартном подходе (для тех случаев, в которых число задач, решаемых при стандартном подходе, было доступно). Помимо этого, навыки обучаемых возрастали в ходе игры, это было видно по положительной динамике затраченных на уровень времени и числа шагов по отношению к сложности уровня. А именно, убыванию с возрастанием уровня отношений времени / числа шагов большинства играющих к хорошему для уровня времени / числу шагов.

Помимо этого, эксперименты показали ряд интересных особенностей:

1. Вредность использования ограничений по времени на уровень при использовании игры в обучающих целях. Данной ограничение тестировалось в эксперименте 1 и приводило к возрастанию применения неоптимальных правил с большой интенсивностью. То есть обучаемые переходили к случайному подбору правил при приближении временных границ.

2. Недостаточность визуальных объяснений для самостоятельного вникания в суть игры. А именно, многие из школьников 10-11 классов бросали игру, не решив даже вводной задачи, которая решается за один шаг.

3. Сложность продумывания качественных игр и недостаток удобных инструментов для их самостоятельного создания преподавателями.

\section{Зак^ючение}

Предложенный способ игрофикации процесса решения учебных задач на преобразование символьных выражений позволяет стимулировать учащихся к решению существенно большего числа задач на применение формул без существенного увеличения временных затрат со стороны преподавателей. Это позволяет повысить навыки учащихся по владению формулами, и, таким образом, способствует повышению качества образования в целом. В текущий период вынужденной самоизоляции предлагаемые способы становятся особенно актуальны. 
Результаты использования метода зависят от качества подготовки уровней-задач, их количества и комбинации с другими теоретическими и практическими упражнениями. Текущие способы настройки уровней слишком сложны для широкого применения. Мы про- должаем работать в этой области, наши дальнейшие планы связаны с созданием удобных инструментов для конфигурирования игр, возможности автоматической генерации качественных задач-уровней и продумыванием новых способов игрофикации.

\section{ЛИТЕРАТУРА}

1. Auvinen T. Harmful Study Habits in Online Learning Environments with Automatic Assessment. Proceedings of the 2015 International Conference on Learning and Teaching in Computing and Engineering. - 2015 - C. 50-57.

2. Willman S., Linden R., Kaila E., Rajala T. and Laakso M. On study habits on an introductory course on programming. Computer Science Education. - 2015.C. 276-291.

3. Tirronen M. and Tirronen V. A framework for evaluating student interaction with automatically assessed exercises. Koli Calling '16 Proceedings of the 16 th Koli Calling International Conference on Computing Education Research.— 2016.—C. 180-181.

4. Gettinger, M., and Seibert, J. K. Best practices in increasing academic learning time. Best pract. Sch. Psychol. IV 1. — 2002 — P. 773-787.

5. Marsh, H. W., and Ware, J. E. Effects of expressiveness, content coverage, and incentive on multidimensional student rating scales: new interpretations of the dr. fox effect. J. Educ. Psychol. - 1982 - P. 74, 126.

6. Bretzke, H., and Vassileva, J. (2003). "Motivating cooperation on peer to peer networks," in 9th International Conference, UM 2003, eds P. Brusilovski, A. Corbett, F. de Rosis (Johnstown, PA).

7. Boud, D., and Molloy, E. (2013). Feedback in Higher and Professional Education: Understanding It and Doing It Well. London: Routledge.

8. Coursera. [Электронный ресурс] — Режим доступа: https://ru.coursera.org/

9. EDX. [Электронный ресурс] — Режим доступа: https://www.edx.org/. Последний доступ 05.08.2020.

10. Stepik. [Электронный ресурс] — Режим доступа: https://welcome.stepik.org/en.

11. WeBWork. [Электронный ресурс] — Режим доступа: https://webwork.elearning.ubc.ca/webwork2/.

12. Moodle. [Электронный ресурс] — Режим доступа: https://moodle.org/.

13. Kahoot. [Электронный ресурс] — Режим доступа: https://kahoot.com/.

14. Castle Quiz. [Электронный ресурс] — Режим доступа: https://clevver.me/.

15. Euclidea. [Электронный ресурс] — Режим доступа: https://www.euclidea.xyz/.

16. Пифагория. [Электронный ресурс] — Режим доступа: https://play.google.com/store/apps/details?id=com.hil_hk.pythagorea/.

17. XSection. [Электронный ресурс] — Режим доступа: https://play.google.com/store/apps/details?id=com.hil_hk.xsection/.

18. Обзор игр на интересную геометрию. [Электронный ресурс] — Режим доступа: https://helpix.ru/appinion/201612/1831-pifagorija_euclidea-interesnaja_ geometrija.html/

19. GeoGebra. [Электронный ресурс] — Режим доступа: https://www.geogebra.org/.

20. Novikov F. and Katsman V. Gamification of Problem Solving Process based on Logical Rules. Springer LNSC. — 2018 — C. 369-380,

21. Новиков Ф.А., Кацман В. И. Автоматическая проверка решений учебных задач на основе комбинации методов перебора логических правил и тестирования. Цифровые технологии в инженерном образовании: новые тренды и опыт внедрения (IT-Technologies for engineering education: new trends and implementation experience), Москва: Издательство МГтУ им. Н. Э. Баумана, — $2020-$ C. 266-273.

22. Реализованное нами Android-приложение Мatify. [Электронный ресурс] — Режим доступа: https://play.google.com/store/apps/details?id=mathhelper. games.matify/.

(c) Кацман Виктор Игоревич ( vikto9494@gmail.com ), Козлов Илья Алексеевич ( kozlovilya@bk.ru ),

Новиков Федор Александрович ( fedornovikov51@gmail.com ).

Журнал «Современная наука: актуальные проблемы теории и практики» 